\title{
Philosophiques
}

\section{D’une définition herméneutique de la métaphysique}

\section{François Jaran}

Volume 41, numéro 2, automne 2014

URI : https://id.erudit.org/iderudit/1027227ar

DOI : https://doi.org/10.7202/1027227ar

Aller au sommaire du numéro

Éditeur(s)

Société de philosophie du Québec

ISSN

0316-2923 (imprimé)

1492-1391 (numérique)

Découvrir la revue

Citer ce document

Jaran, F. (2014). D’une définition herméneutique de la métaphysique.

Philosophiques, 41(2), 379-385. https://doi.org/10.7202/1027227ar d'utilisation que vous pouvez consulter en ligne.

https://apropos.erudit.org/fr/usagers/politique-dutilisation/ 


\title{
D'une définition herméneutique de la métaphysique
}

\author{
FRANÇOIS JARAN \\ francoisjaran@gmail.com \\ Universitat de València, Espagne
}

L'ouvrage de Jean Grondin, Du sens des choses. L'idée de la métaphysique, cherche à souligner le caractère éminemment métaphysique de la pensée herméneutique dans ce que l'on peut considérer comme une apologie du trait métaphysique de toute la pensée philosophique. Il s'agit donc pour l'auteur de défendre, dans un premier temps, l'intérêt actuel pour la pensée métaphysique qui a été malmenée par quelques décennies de dépassements et de déconstructions. Bien que la "renaissance " actuelle de la métaphysique qui se généralise même là où l'on devrait le moins s'y attendre, dans la tradition analytique - semble présager des temps plus cléments pour la science de l'étant en tant qu'étant, cela reste encore un enjeu philosophique d'importance que de démontrer la légitimité philosophique de la «reine des sciences". Dans un deuxième temps, il s'agit aussi de montrer que la métaphysique est une pensée éminemment herméneutique. Bien que ce lien «naturel » ait plutôt été décrié (Rorty, Vattimo) qu'encensé, c'est bien l'intention de l'auteur que de montrer que l'herméneutique n'est pas une critique de la métaphysique mais bien sa plus récente refondation; mais aussi et surtout que la métaphysique ne peut se faire sans herméneutique. Afin d'élucider cette thèse - plutôt forte même si l'auteur la présente comme une évidence - , nous proposons de nous enquérir à même l'ouvrage de Jean Grondin de ce qu'est la métaphysique.

Nous connaissons déjà les réponses traditionnelles (d'Aristote à Heidegger) à la question Qu'est-ce que la métaphysique? mais nous aimerions chercher quelle définition, quelle intelligence de la métaphysique émerge d'une lecture attentive de l'ouvrage. Le problème ici évoqué rappelle celui de la pluralité de la métaphysique dont parle Jean Grondin dans la première de ses leçons (p. 3-4). Est-il légitime en effet de parler de La métaphysique, c'est-à-dire d'une tradition unique qui se serait vouée aux questions que l'on dit métaphysiques? Y a-t-il suffisamment de ressemblances entre Platon, Aristote, Thomas d'Aquin, Descartes, Kant et Heidegger pour affirmer que chacun d'eux s'est consacré à la métaphysique? La tentation est grande aujourd'hui de répondre par la négative: ce qu'entendraient tous ces penseurs par métaphysique se ressemble si peu que c'est une illusion (claironnée par Heidegger) que de parler de $L a$ métaphysique ${ }^{1}$. Pourtant, la facilité avec

1. Un ouvrage intitulé Y a-t-il une histoire de la métaphysique? (Y.-C. Zarka et B. Pinchard (dir.), PUF, 2005) s'est d'ailleurs récemment penché sur la question.

PHILOSOPHIQUES 41/2 - Automne 2014, p. 379-385 
laquelle on reconnaît les métaphysiciens ne trompe pas: la métaphysique est sans doute difficile à définir, mais elle est bien une façon reconnaissable de questionner. On ne la confond jamais avec la gastronomie ou l'art équestre, par exemple, et on sait d'elle qu'elle prétend dire ce qu'il en est des questions humaines dernières - «dernières" signifiant ici du même coup «les plus générales» et "les plus élevées". Ici, c'est comme l'effort inévitable de comprendre la transcendance derrière l'immanence que Jean Grondin cherchera à définir la métaphysique.

Le thème qui nous intéresse est traité de façon explicite aux deux derniers chapitres, dans la sixième leçon et l'épilogue, mais hante l'ouvrage au complet. Car, dès la première page, la question du sens à donner à l'expression «métaphysique» fait surface lorsque l'auteur annonce qu'il s'efforcera de la présenter "comme une écoute du sens des choses» (p. v). Le mot chose va évidemment toujours de pair avec la métaphysique, mais cela est moins vrai du mot sens, et sans doute encore moins du mot écoute. Le trait passif, serein, de l'écoute contraste bien sûr avec le caractère supposément violent, dominateur que l'on a longtemps cherché à associer à cette pensée qui, selon Heidegger, est si éloignée de la sérénité, de la Gelassenheit, qu'elle ne laisserait même pas l'arbre être "debout là où il est $^{2}$ ». Mais puisque c'est du caractère herméneutique de la métaphysique qu'il est ici question, c'est sans nul doute au mot sens qu'il faut le plus faire attention.

C'est en effet d'une herméneutique métaphysique - et non plus «simplement» d'une herméneutique philosophique, comme le souhaitait Gadamer - que se réclame Jean Grondin. Celle-ci se fonderait sur l'idée que "l'homme est un être de compréhension et que ce qu'il cherche à comprendre, c'est le sens des choses» (p. vi). Certes, cette définition de l'homme ou de l'existence humaine, à partir de la compréhension qu'il a des choses, est précisément ce qui a permis à l'herméneutique, à partir de celle de la «factiticité » élaborée par Heidegger au début des années I920, de devenir un courant philosophique au $\mathrm{xx}^{\mathrm{e}}$ siècle. Si la philosophie doit se convertir à l'herméneutique, soutient Heidegger dans ses écrits herméneutiques, c'est tout simplement parce que l'homme est un être voué à l'interprétation et à la compréhension, un animal hermeneuticum. Or cette thèse au sujet de la nature humaine n'est pas déjà une thèse métaphysique, dans la mesure où Heidegger ne conçoit pas encore la philosophie comme une entreprise vouée à une tâche ontologique - celle de répondre à la question de l'être, comme ce sera le cas dans Sein und Zeit - mais bien comme une tentative dont l'objet ultime est la vie humaine saisie dans sa facticité. Dans sa face la plus visible, l'herméneutique de Gadamer suit ces directives. Cependant, comme le montre bien Jean Grondin, Wahrheit und Methode caressait aussi de façon discrète des ambitions métaphysiques. Mais qu'est-ce qui distingue exactement l'herméneutique métaphysique de l'herméneutique philosophique?

2. Was heißt Denken? (GA 8), p. 46; trad. fr. Qu'appelle-t-on penser?, p. 45 . 
Dans l'avant-propos de l'ouvrage, Jean Grondin présente ce caractère herméneutique de la métaphysique - non seulement de sa propre métaphysique, mais bien de toute métaphysique - ainsi que le caractère métaphysique de toute herméneutique comme une évidence. Tout d'abord, une métaphysique ne peut se déployer "sans mettre en œuvre une interprétation". Ensuite, "une herméneutique ne peut pas ne pas être métaphysique au double sens où ce qu'elle pense est quelque chose qui est et où ce qui est alors compris l'est nécessairement à la lumière de son sens, qui à la fois l'enveloppe et le dépasse » (p. vi). Si c'est surtout le caractère herméneutique de la métaphysique qui intéresse l'auteur et nous, une remarque s'impose sur le caractère nécessairement métaphysique de l'herméneutique. Bien sûr, il faut voir ici une prise de position contre l'association qui semble aller de soi entre herméneutique et dépassement de la métaphysique (voir à ce sujet l'épilogue), mais on peut quand même se demander si l'argument est adéquat. Tout d'abord, que l'herméneutique ait pour objet de pensée des choses qui sont ne fait évidemment pas d'elle une métaphysique. Toute science à cet égard serait aussi une métaphysique. Cependant, la métaphysique, du moins comme métaphysique générale, se caractérise négativement par rapport aux sciences particulières qui, précisément, ne traitent jamais que de choses qui sont plutôt que du fait que les choses soient. Mais il faut lire la phrase au complet: ce que l'herméneutique pense, elle le comprend à la lumière de son sens. C'est donc le sens qui donnerait à l'herméneutique son caractère métaphysique dans la mesure où rien ne peut être compris qui ne soit sens. L'universalité de l'herméneutique - à laquelle Jean Grondin a d'ailleurs consacré un ouvrage important $t^{3}-$ serait donc ce qui lui confère son caractère métaphysique: puisque l'existence n'aurait jamais affaire qu'à des choses qui se présentent à la lumière de leur sens, l'interprétation et la compréhension constitueraient cet horizon à partir duquel le monde se manifeste et dont la mise en lumière constituerait la tâche spécifique de la métaphysique.

Or cela ne peut être soutenu que si l'on accepte cette identification de la chose ou de l'étant au sens de la chose ou de l'étant. C'est d'ailleurs ce que Jean Grondin prétend faire tout au long de l'ouvrage, c'est-à-dire briser la conception d'origine sans doute kantienne (p. I०8) selon laquelle nous avons accès au monde à travers nos facultés dont font partie l'interprétation et la compréhension. Dans cette optique, le monde et les choses ne seraient jamais saisis qu'au travers de notre capacité à donner du sens au monde et aux choses. C'est cette idée qui est ici attaquée au profit d'une nouvelle intelligence de notre relation au monde et aux choses: le monde et les choses sont leur sens, se donnent toujours comme sens. La saisie du sens serait une saisie perceptive et non une activité de second ordre qui réinterprèterait à sa façon les choses mêmes, initialement données sans sens. Or cette herméneutique métaphysique cherche à justifier sa nature à partir d'une définition précise de 
l'essence de la métaphysique. Pour comprendre cette idée, il est donc fondamental de nous arrêter sur ce qu'entend l'auteur par "métaphysique».

La métaphysique est inévitable. La première idée fondamentale que présente l'auteur est celle de la présence nécessaire de la métaphysique, c'està-dire celle d'une métaphysique implicite, inévitable. Même si l'on souhaite s'en défaire, la métaphysique rattrape toujours la philosophie, non pas tant comme intérêt propre de la raison humaine mais surtout parce que toute critique de la métaphysique aurait toujours ses propres présupposés métaphysiques. Selon ce que défend Jean Grondin, on ne dépasse jamais la métaphysique qu'au nom d'une autre métaphysique (p. 6). Cela serait particulièrement vrai pour ces penseurs qui ont cherché à dépasser la métaphysique pour mieux répondre à ses questions propres, comme c'est le cas de Heidegger ou encore de Levinas, qui substitue l'éthique à l'ontologie tout en conservant le schéma de la philosophie première. La métaphysique serait donc l'arrière-fond de toute la pensée, les fondations de toute recherche de sens.

L'auteur emploie cette idée non seulement pour critiquer la volonté déconstructrice de la pensée philosophique qui prétend critiquer la métaphysique, mais aussi pour montrer que les philosophes qui ont cherché à prendre leurs distances, voire à ignorer la métaphysique, ne sont en réalité que des métaphysiciens qui s'ignorent. Le nominalisme - c'est l'exemple que l'auteur emploie le plus souvent - ne serait en fin de compte qu'une métaphysique, assez rudimentaire de surcroît: celle qui "ne tient pour réel que ce qui bénéficie d'une existence individuelle susceptible d'être observée " (p. I 3 I $)^{4}$. Selon cette critique des pensées qui pensent s'émanciper de la métaphysique, toute affirmation sur ce qui est constituerait nécessairement une «rechute» — ou plutôt un «maintien» puisqu'on n'en sort jamais dans la métaphysique.

La métaphysique est un effort inévitable de compréhension. Cette seconde idée définit la métaphysique comme «l'effort vigilant de la pensée

4. Cette tendance "continentale», peut-être même heideggérienne en fin de compte, à considérer que la tradition empiriste - et sa tendance nominaliste - a elle-même cherché à s'exclure de l'histoire de la métaphysique est sans aucun doute moins juste que l'on pourrait le croire. Après tout, Thomas Hobbes est l'un des penseurs invités par Mersenne à participer aux Disputationes entourant la publication des Méditations de Descartes. Dans ses objections, Hobbes ne nie aucunement l'existence et la pertinence des questions métaphysiques, mais propose plutôt une autre métaphysique, plus adéquate, celle qui nie la capacité de penser quelque chose qui ne soit pas corporel (Meditationes de Prima Philosophia, Objectiones Tertiae, AT VII, p. I73). De la même manière, les problèmes que pose David Hume ne sont pas seulement métaphysiques rétrospectivement, c'est-à-dire parce que Kant les aurait considérés tels, mais le sont aussi dans le texte même. Dans la première section de An Enquiry Concerning Human Understanding, Hume inscrit ouvertement son essai sous l'égide de la métaphysique et affirme comme tout bon défenseur de la pensée métaphysique qu'il nous faut «cultiver avec soin la véritable métaphysique, afin de détruire la métaphysique fausse et dénaturée » ("Section I: On the Different Species of Philosophy»). 
humaine de comprendre l'ensemble de la réalité et ses raisons» (p. 2I). Comprendre la réalité et ses principes, c'est bien la tâche que l'on identifie comme celle de la philosophie et, plus spécifiquement, comme celle de la métaphysique. Il faut noter cependant que l'auteur insiste sur l'idée d'un effort, d'un exercice, et aussi sur l'aspect dialogique (p. 24) de la métaphysique et non, comme ce serait le cas d'une définition plus classique, sur sa nature de "discours» ou de "science». Elle est plutôt une recherche de sens qui peut toujours se solder par un échec. Elle est une tentative de répondre aux questions dernières, une tentative qui n'établit jamais d'avance les conditions de son succès (comme ce peut être le cas de la science) dans la mesure où elle ne cherche pas tant à expliquer qu'à comprendre. Ainsi, la métaphysique n'est précisément pas cette volonté inquisitrice et violente qui chercherait à donner une explication définitive de la réalité.

La métaphysique est l'effort inévitable de comprendre la transcendance derrière l'immanence. La seconde définition de la métaphysique est associée immédiatement à une troisième, celle d'une recherche de transcendance derrière l'immanence. L'auteur convoque à cet effet Dilthey pour qui comprendre consiste toujours en un effort " de percer l'intériorité des choses, tâcher de deviner le secret qui se cache derrière leur manifestation, entendre le verbe intérieur, non dit, en amont de ce qui est proféré» (p. 28). Alors que l'explication demeurerait à la surface des choses, c'est-à-dire au niveau de la manifestation, la compréhension qui caractérise la tâche de la métaphysique serait toujours à la recherche des motifs cachés derrière les manifestations. On comprend les larmes qui coulent sur les joues d'un enfant quand on découvre les motifs qui ont donné lieu à ces pleurs. Or ces motifs ne se donnent jamais qu'au travers de manifestations particulières, et c'est à l'interprète qu'il appartient de les reconnaître. Cela est certainement ainsi dans le monde de l'esprit. L'action humaine n'est en effet véritablement saisie que lorsqu'elle est comprise. Mais peut-on appliquer ce schéma à toute chose? Selon l'auteur, le même schéma peut être employé pour ce qui est du fondement de toute métaphysique qu'est l'apparence visible des choses (p. 43). Par l'intelligence humaine, un second ordre des choses apparaît nécessairement, de telle sorte qu'advient ce que l'on appelle la métaphysique. Ce second ordre ne correspondrait pas à cela qui ne se voit ou ne se montre pas, mais bien à cela qui, pour être vu, exige un "second regard ", une "distance par rapport à la première apparence des choses» (p. I 22).

C'est cette idée d'une recherche de transcendance qui va animer la dernière leçon: "La meilleure métaphysique, celle qui prend au sérieux l'intelligence des choses, est celle qui pressent que l'immanence laisse deviner quelque transcendance et que la transcendance n'en est pas une si elle n'investit pas l'immanence» (p. I26). Le rôle de la métaphysique ne serait plus ici seulement de poser les questions les plus générales et les plus éminentes de la philosophie, mais aussi de prendre position sur la réalité du réel de telle sorte que devienne possible la compréhension de ce qui, de façon immédiate, 
n'apparaît pas. La métaphysique a en effet ici pour tâche de s'interroger de façon herméneutique - sur la justification de l'affirmation selon laquelle "la réalité immédiatement visible, sensible, temporelle, n'est peut-être pas la seule» (p. I29).

Ainsi, nous avons identifié trois éléments fondamentaux de la définition que donne Jean Grondin de la pensée métaphysique: (I) quoi qu'il fasse, le philosophe ne peut nier que sa pensée comporte une dimension métaphysique, puisque (2) toute pensée philosophique s'efforce toujours de comprendre la réalité, le monde, la vérité, etc., ce qui veut nécessairement dire (3) chercher cela qui se tient au fondement de la réalité sensible.

Or on doit s'interroger sur l'unité de la définition de la métaphysique présentée ici, plus précisément sur la co-appartenance des premier et troisième éléments. En effet, l'idée d'une métaphysique implicite suppose que toute pensée philosophique, c'est-à-dire même la pensée nominaliste et matérialiste qui identifie la réalité à ce qui peut être observé à première vue, soit tenue même contre son gré pour une pensée métaphysique. Pourtant, l'association que présente l'auteur entre la métaphysique et l'idée de transcendance semble renier cette thèse dans la mesure où elle exclut la possibilité d'une métaphysique de l'immanence comme l'est l'explication matérialiste de la réalité qui se déploie à partir des seuls concepts de matière et de hasard. Si la métaphysique est cette pensée qui cherche à transcender l'aspect immanent des choses, toute pensée qui refuse de le faire ne devrait plus être considérée comme une métaphysique qui s'ignore, mais bien comme une pensée non métaphysique, voire antimétaphysique. Cela est cependant interdit par la première idée, celle selon laquelle toute pensée philosophique est au moins secrètement une métaphysique. Toute philosophie, volens nolens, se prononce toujours sur la réalité du réel, sur l'être de l'étant, mais cela implique$\mathrm{t}$-il que toute philosophie admette la transcendance?

On peut cependant se demander s'il est vrai que toute philosophie doive nécessairement se prononcer sur la réalité du réel. N'existe-t-il pas des mécanismes qui permettent à la pensée de reconnaître ce fait et de "courtcircuiter " cette tendance naturelle de la pensée à s'interroger sur l'être des choses? Pour clore ce petit commentaire, nous aimerions évoquer une forme de pensée - pratiquement absente de l'ouvrage de Jean Grondin - qui, à notre humble avis, parvient précisément à éviter de prendre position sur les questions métaphysiques, non pas en les ignorant ou en les critiquant, mais bien plutôt en les identifiant et en se refusant d'y répondre. Cette pensée, qui dans l'ouvrage est accusée d'être une métaphysique qui s'ignore, c'est la phénoménologie de Husserl. Cette pensée reconnaît elle aussi que la philosophie se mêle toujours des affaires de la métaphysique, qu'elle le cherche explicitement ou non. En ce sens, elle s'accorde avec l'idée que toute philosophie contient une métaphysique implicite. Mais du moment qu'elle reconnaît cette particularité de la pensée philosophique et qu'elle rend explicite ce qui n'est jamais qu'implicite, elle peut chercher à déjouer cette tendance de la 
pensée non pas en niant la pertinence des questions métaphysiques - identifiées à celles, aristotéliciennes, d'une « science de l'être au sens absolu ${ }^{5}$ » mais bien en reconnaissant leur fonction et en développant une méthode philosophique, la réduction phénoménologique, qui les mette en suspens. Husserl ne soutiendra jamais - comme tant d'autres - que la métaphysique soit une erreur de la philosophie, mais plutôt que cette tendance à réfléchir sur l'être des choses peut être neutralisée de telle sorte que notre jugement au sujet de ce qui transcende la conscience soit retenu. Ainsi s'élabore une pensée qui n'est pas antimétaphysique, mais bien $a$-métaphysique.

Au sujet de la phénoménologie de Husserl, Jean Grondin note que "parler de ce qui se manifeste à la conscience, c'est parler d'un être qui apparaît et se prononcer sur son être» (p. 5, note I). Cette métaphysique implicite chez Husserl, Heidegger croyait aussi la trouver: son refus de répondre à la question de l'être le forçait à adopter une métaphysique traditionnelle (la cartésienne) et toutes les réponses qui lui sont liées. Mais une lecture plus charitable de Husserl montre qu'il a parfaitement identifié ce qui intéresse la métaphysique - l'existence réelle des choses - et qu'il est convaincu que ces questions ne peuvent se décider que sur une base plus fondamentale qui est celle de l'apparition des choses à la conscience. Cette manifestation des choses, ce n'est pas une façon d'exister, c'est-à-dire une façon d'être indépendante de la conscience du sujet - comme ce sera le cas chez Heidegger pour qui l'apparence est nécessairement liée à l'être: "Wieviel Schein jedoch, so viel Sein ${ }^{6} »-$, mais bien quelque chose qui advient par-delà la question de savoir si les choses existent réellement ou non.

On peut certes reprocher à Husserl de ne pas souscrire à l'idée de la primauté absolue de la question de l'être, mais on ne peut pas l'accuser d'être malgré lui métaphysicien. Husserl sait parfaitement que la pensée philosophique tend vers les questions métaphysiques, mais il croit être capable d'isoler ce qui, dans la pensée philosophique, appartient à la pensée métaphysique et ce qui y échappe. Si cela se peut faire, Husserl serait la preuve que la philosophie est à même de reconnaître ses propres mécanismes métaphysiques et qu'elle peut les neutraliser grâce à l'identification d'un sol plus originaire que l'être et la compréhension qu'on en a: la manifestation.

5. Voir, entre autres, Die Idee der Phänomenologie. Fünf Vorlesungen, dans Husseliana, tome II, La Haye, Martinus Nijhoff, I973, p. 23.

6. Sein und Zeit, Halle an der Saale, Niemeyer, I927, p. 36. 